\title{
FRIEDRICH HAYEK: LA TEORÍA DEL CONOCIMIENTO Y LA FUNDAMENTACIÓN GNOSEOLÓGICA DE LA CRÍTICA A LA ECONOMÍA PLANIFICADA
}

LEANDRO GASTÓN INDAVERA STIEBEN

Universidad Nacional de Chilecito 


\title{
FRIEDRICH HAYEK: LA TEORÍA DEL CONOCIMIENTO Y LA FUNDAMENTACIÓN GNOSEOLÓGICA DE LA CRÍTICA A LA ECONOMÍA PLANIFICADA*
}

\begin{abstract}
Resumen: el objetivo de este trabajo será, en primer lugar, analizar algunos textos de Hayek que den cuenta de sus concepciones sobre el conocimiento y tratar de mostrar cómo dichas concepciones le sirven a Hayek como herramienta para fundamentar en parte su concepción sobre la necesidad del libre mercado como único sistema viable y eficiente de producción y distribución económica en detrimento de la planificación económica centralizada. En segundo lugar, se mostrará cómo esta concepción hayekiana puede servir para interpretar los fundamentos de una teoría subjetiva del valor.

Palabras clave: Hayek, conocimiento, crítica, economía, planificada.

\section{FRIEDRICH HAYEK: THE THEORY OF KNOWLEDGE AND THE EPISTEMIC FOUNDATION FOR THE CRITICISM OF PLANNED ECONOMY}

\begin{abstract}
Firstly, the aim of this paper is to analyse several Hayek's writings in order to understand his conception of knowledge and in order to show how these conceptions are useful as a tool to set, partially, the foundations of free market and the denial of any economic planning. Secondly, Hayek's ideas will be analysed in order to show how it is possible to build a subjective theory of value based on Hayek's theory of knowledge.
\end{abstract}

Keywords: Hayek, knowledge, criticism, planned, economy.

Fecha de recepción: Junio 16 de 2015

Fecha de aceptación: Junio 15 de 2016

Forma de citar (APA): Indavera, L. (2016). Friedrich Hayek: la teoría del conocimiento y la fundamentación gnoseológica de la crítica a la economía planificada. Revista Filosofía UIS, 15 (1), 89-105, doi: http://dx.doi.org/10.18273/revfil.v15n1-2016004

Forma de citar (Harvard): Indavera, L. (2016). Friedrich Hayek: la teoría del conocimiento y la fundamentación gnoseológica de la crítica a la economía planificada. Revista Filosofía UIS, 15 (1), 89-105.

Leandro Gastón Indavera Stieben: argentino. Profesor de la Universidad Nacional de Chilecito. Doctor en Epistemología e Historia de la Ciencia de la Universidad Nacional Tres de Febrero.

Correo electrónico: leaindavera@hotmail.com

*Artículo de reflexión.

Revista Filosofía UIS, Vol. 15, No. 1, enero-junio de 2016 pp. 89-105 


\section{FRIEDRICH HAYEK: LA TEORÍA DEL CONOCIMIENTO Y LA FUNDAMENTACIÓN GNOSEOLÓGICA DE LA CRÍTICA A LA ECONOMÍA PLANIFICADA}

\section{Introducción}

Hayek es un autor que trae polémica. No sólo hoy, sino también en su época, entre sus contemporáneos. En nuestros días, Hayek es atacado por sus concepciones metodológicas desde el paradigma neoclásico, mainstream, de la metodología económica académica contemporánea. Hasta algunos liberales que comparten sus ideas políticas, en el ámbito metodológico critican las diferentes posturas hayekianas y ven con condescendencia y desprecio a la escuela austríaca; escuela que tiene a Hayek como uno de sus principales exponentes (Luján, 2003, p.37). Sin embargo, Hayek es también criticado por las ideas que comparte en común con el paradigma metodológico neoclásico; a saber, el supuesto de competencia perfecta y por consiguiente una defensa del sistema del libre mercado desde la teoría económica. Más aún, Hayek es considerado como el mentor teórico del neoliberalismo por muchos autores, hasta tal punto que Gómez (2003) marca el año 1944 como crucial para el inicio de lo que comúnmente denominamos Neoliberalismo, con la publicación de Hayek: Camino de servidumbre (2005) El hecho es que no sólo la posición de Hayek se centra en un análisis estrictamente económico; sino que se despliega por un sinnúmero de campos del saber, pasando por la Economía, la Sociología, la Ciencia Política, la Filosofía, la Metodología de la Ciencia y hasta aún por la Psicología. De este modo, su empeño consistente en elaborar una defensa del sistema de libre mercado y del capitalismo llega hasta la discusión del fin de la historia y la consecuente victoria del sistema capitalista por sobre otros sistemas de producción y distribución económica.

Pero Hayek no solamente hoy en día es polémico. Entre sus contemporáneos mantuvo discusiones metodológicas con los representantes de la escuela neoclásica y la escuela histórica alemana y discusiones políticas en torno a las ventajas y desventajas de un sistema económico planificado. Si en nuestros días (más precisamente a partir de la década de los años setenta con el avance de los llamados "tigres asiáticos" y fundamentalmente a partir de los años noventa con el consenso de Washington y la aplicación de políticas neoliberales en todo el globo 
y más profundamente en Latinoamérica) el paradigma generalmente aceptado en el mundo económico académico en el ámbito de las políticas económicas es el libre mercado y la descentralización de las decisiones económicas, en la época de Hayek era diferente. Fundamentalmente a partir de la década del treinta, debido al crac del veintinueve y a la Gran Depresión, la tendencia predominante en los ámbitos económicos académicos y en las políticas públicas fue una cada vez más fuerte intervención del Estado en las decisiones económicas nacionales y una preocupación cada vez más acentuada en cómo planificar la actividad económica. Hayek, defensor a ultranza de los principios de libre mercado, era una de las excepciones en un ámbito político-económico cada vez más preocupado por los procesos de planificación central y elaboración de planes quinquenales; aún en países capitalistas como Gran Bretaña. Además, más allá de la tendencia en política económica a partir de los años treinta, los contemporáneos de Hayek en Gran Bretaña (académicos o no, como cuenta el mismo Hayek) veían con no muy buenos ojos las consecuencias políticas de un capitalismo de libre mercado; a saber, el avance del Nacionalsocialismo como una respuesta al socialismo (Hayek 1994, p. 102).

El objetivo de este trabajo será, en primer lugar, analizar algunos textos de Hayek que den cuenta de sus concepciones sobre el conocimiento y tratar de mostrar cómo dichas concepciones le sirven a Hayek como herramienta para fundamentar en parte su concepción sobre la necesidad del libre mercado como único sistema viable y eficiente de producción y distribución económica en detrimento de la planificación económica centralizada. En segundo lugar, se mostrará cómo esta concepción hayekiana puede servir para interpretar los fundamentos de una teoría subjetiva del valor.

\section{2. "Economics and Knowledge": el comienzo del interés de Hayek por la teoría del conocimiento}

Terence Hutchison, uno de los metodólogos de la Economía más importantes del siglo XX, sostiene que Hayek realizó un giro metodológico de ciento ochenta grados a mediados de la década de 1930 con su artículo: "Economics and Knowledge"; de sostener las posiciones metodológicas de Ludwig Von Mises sobre la teoría económica a sostener las de Karl Raymund Popper. ${ }^{1}$ La posición metodológica de Mises (uno de los representantes de la escuela austriaca junto con Hayek, Menger y Böhm-Bawerk), que se oponía al Neopositivismo, al Empirismo y fundamentalmente a la escuela histórica alemana, sostenía principalmente que la teoría económica se constituye de enunciados a priori que, aunque diferentes

\footnotetext{
1 Se recomienda consultar Hutchison, 1981, Capítulo VII. Scarano también sostiene esta posición, indicando que Hayek no estaba de acuerdo con el apriorismo miseano (Scarano, 2004, pp.11-12).
} 

gnoseológica de la crítica a la economía planificada

de los de la Lógica y la Matemática, no pueden refutarse empíricamente. ${ }^{2}$ De este modo, un giro de Mises a Popper implicaba para Hayek el paso de una concepción apriorística de los postulados de la Economía a una concepción que, en consonancia con el falsacionismo de Popper, sostuviese la posibilidad de refutación de los enunciados de la Economía.

Pero, independientemente de este cambio metodológico, "Economics and Knowledge" es importante en el análisis de la obra de Hayek porque, como el mismo Hayek contaría años más tarde, a partir de la publicación de dicho artículo en 1936 él comienza a interesarse por la investigación sobre cuestiones que van más allá de la pura teoría económica o las técnicas derivadas de ésta:

"Though at one time a very pure and narrow economic theorist, I was led from technical economics into all kinds of questions usually regarded as philosophical. When I look back, it seems to have all begun, nearly thirty years ago, with an essay on "Economics and Knowledge" in which I examined what seemed to me some of the central difficulties of pure economic theory [...]" (1967, p. 91).

En efecto, "Economics and Knowledge" inauguró una serie de investigaciones interdisciplinarias, que sobrepasan la frontera del análisis estrictamente económico. En las primeras líneas del artículo encontramos la fundamentación de por qué Hutchison sostiene que Hayek da un giro de ciento ochenta grados en cuanto a posiciones metodológicas se refiere. Allí, el mismo Hayek sostiene que la teoría económica tiene un elemento empírico que es pasible de ser falsado (el mismo Hayek cita a Logik der Forschung de Popper cuando utiliza el concepto de falsación); a saber: las proposiciones acerca de la adquisición del conocimiento. En efecto, lo que se propondrá Hayek en "Economics and Knowledge" es ver qué papel juegan las proposiciones sobre el conocimiento que los agentes económicos poseen en el análisis económico (Hayek, 1980a, p. 33). Este artículo también es el comienzo de una serie de críticas de Hayek dirigidas a quienes conciben el análisis del equilibrio general macroeconómico como simplemente un sistema lógico formal que no debe someterse a contrastación empírica, del mismo modo que sucede con la matemática o la geometría. Más bien, según Hayek, el estudio del análisis del equilibrio general debe contener no proposiciones tautológicas; sino proposiciones empíricas basadas en cómo el conocimiento es adquirido y comunicado (33-35).

La escuela económica que está criticando Hayek en el mencionado artículo, al hacer una crítica del tratamiento formal del equilibrio económico general, es la tradición neoclásica iniciada principalmente por Jevons, Walras y Menger. La escuela neoclásica es la tendencia principal, o mainstream, del mundo académico

\footnotetext{
${ }^{2}$ Se recomienda consultar Scarano, 2004, pp.1- 9. Sobre las relaciones entre en apriorismo de Hayek y el kantiano ver también Connin, 1990, p.302 y Horwitz, 1990.
} 
económico de nuestros días. Si bien se asocia a esta escuela con el ámbito de las políticas económicas liberales, de las cuales Hayek es un gran defensor y mentor teórico, existe una diferencia fundamental entre los postulados metodológicos y las concepciones sobre el agente económico entre la escuela neoclásica y la escuela austriaca (entre cuyos principales exponentes encontramos a Hayek). Una de las diferencias fundamentales, como ya se mencionó sucintamente, es la aceptación o no de los enunciados del análisis del equilibrio económico. Pero la diferencia que más nos interesa a nosotros con relación a las concepciones sobre el conocimiento de Hayek es la que versa sobre el grado de completitud del conocimiento del agente económico.

En la teoría económica de la escuela neoclásica, el agente económico tiene información completa sobre absolutamente todo el proceso económico y sus preferencias son completas (esto quiere decir que dados dos objetos x o y, el individuo racional elige o bien $x$ por sobre $y, y$ por sobre $x$ o es indiferente frente a dichos objetos) y transitivas (en el sentido lógico que comúnmente es usado el término) (Hausman, 1992, pp. 13-19). Hasta el día de hoy esta es la concepción generalizada de la mayoría de los esquemas microeconómicos. Sin caer en exageraciones, el paradigma neoclásico ve a los agentes económicos como omniscientes en cuanto a sus decisiones económicas (Lawson, 2005 p. 429). Justamente la idea de Hayek en "Economics and Knowledge" es atacar este supuesto de omnisciencia de los agentes económicos racionales. Pero no sólo eso. Lo que más nos interesa específicamente es el ataque que hace Hayek al supuesto neoclásico de que, como los datos de los agentes económicos en cuanto a esquemas de demanda, gustos individuales, hechos técnicos, etc. son igualmente dados a todos los individuos, entonces el equilibrio general macroeconómico se produce porque los planes isomorfos de cada uno de los individuos se adaptarán el uno al otro (Hayek 1980a, p. 38). Hayek sostendrá, en cambio, que el conocimiento económico esta disperso en los individuos; es decir, no todos tenemos acceso a la misma información económica, y mucho menos a toda. El conocimiento económico es, para Hayek, local y sólo tiene sentido en tiempos y lugares determinados (Ortiz, 2009, p. 188).

Hayek establece una distinción, a diferencia de los teóricos neoclásicos, entre dos tipos de datos económicos. Mientras que la teoría del equilibrio como mero sistema lógico establece una relación de equivalencia entre los datos objetivos de la economía (es decir, lo que realmente sucede en el mundo económico) y los datos subjetivos de los agentes económicos (es decir, lo que los agentes económicos piensan que sucede en el mundo económico), Hayek dice que ambos conceptos son diferentes (1980a, p. 39). No necesariamente tienen los datos objetivos de la economía que coincidir con los datos subjetivos de los agentes económicos. Más bien, ambos tipos de datos corresponden a dos niveles de análisis diferentes. Por 
ende, como no es necesario que coincidan, Hayek concluye que: "The equilibrium relationships cannot be deduced merely from the objective facts, since the analysis of what people will do can start only from what is known to them" (1980a, p.44).

Pero dada la cita anterior de Hayek, ¿qué significa hablar de una "tendencia hacia el equilibrio" en la teoría económica? Al no existir el supuesto de equivalencia entre los datos de la actividad económica real y los datos de los agentes económicos, significa que bajo ciertas condiciones "the knowledge and intentions of the different members of society are supposed to come more and more into agreement... that the expectations of the people and... the entrepreneurs will become more and more correct" (45). De este modo, Hayek cree haber dado un giro trascendental en la teoría económica; a saber, haber redescubierto un concepto que había sido negado en la historia del pensamiento económico: la división del conocimiento (50). Así, Hayek sostendrá que el problema principal que la Economía debe resolver es cómo interacciones espontáneas de un número de personas puede llevar hacia un equilibrio general de la economía.

En "The Use of knowledge in Society", Hayek utiliza los conceptos de "Economics and Knowledge" para aplicarlos a su disputa con las concepciones de la economía planificada. Si el conocimiento nunca está concentrado en una sola persona; sino que es disperso, incompleto y contradictorio, el problema económico principal no es al que diera lugar Hume mostrando que los recursos económicos son escasos, ${ }^{3}$ sino que, más bien, el problema principal que debe abordar la economía según Hayek es cómo gestionar y utilizar lo más eficientemente posible un conocimiento sobre la realidad económica que está disperso, y que no está disponible para todos y cada uno de nosotros en su totalidad. ${ }^{4}$ De este modo, si estamos frente al dilema de elegir entre una economía de mercado y una economía planificada, el criterio de elección será aquel sistema que asegure una mejor utilización del conocimiento disperso. Los teóricos de la planificación se basan sobre este punto fundamentalmente, según Hayek, en sostener que si de conocimiento se trata, los expertos científicos están mejor capacitados para tomar las decisiones económicas por todos nosotros. Por ende, el mejor sistema para gestionar conocimientos radica en una planificación científica de nuestras actividades y elecciones económicas. Independientemente del escollo que significa elegir a los expertos científicos, la crítica de Hayek se centra en que el conocimiento científico no es el único existente; sino solamente una parte de él. Justamente, las decisiones económicas no se basan en conocimientos de teorías generales sobre el mundo. Más bien,

\footnotetext{
${ }^{3}$ La referencia a Hume la tomo de Janina Rosicka, quien sostiene que Hume hizo hincapié en la escasez a la hora del análisis económico, y esto dio pie al inicio de la Economía clásica, ya que antes pensar en la Economía suponía concebir una plenitud de recursos (Rosicka, 2002, pp.324-325).

${ }^{4}$ Sobre un análisis comparado sobre las concepciones de Hayek y Mises en relación al cálculo y al conocimiento ver Yeager, 1994.
} 
las decisiones económicas se basan simplemente en el conocimiento de ciertas informaciones particulares en el tiempo y en el espacio. De este modo, sugiere Hayek, la decisión última de la actividad económica debe ser dejada a quien tiene el conocimiento de dicha información particular (Hayek, 1980b, pp. 77-84). Por ende, "in a system in which the knowledge of the relevant facts is dispersed among many people, prices can act to co - ordinate the separate actions of different people in the same way as subjective values help the individual to co-ordinate the parts of his plan" (85).

\section{El proyecto sobre el Abuso de la Razón. El artículo "Scientism"}

En aras de una crítica teórica a la planificación económica y a la centralización de las decisiones económicas que estaban en boga en su época, Hayek emprende un camino de redacción cuyo fruto será el artículo "Scientism" y los libros The Road to Serfdom, The Constitution of Liberty y Law, Legislation and Liberty (Caldwell, 2004, pp.256).

El artículo "Scientism", que luego formará parte de The Counter-Revolution of Science, comienza con una crítica a la pretensión metodológica consistente en aplicar estrictamente la metodología de las ciencias naturales a las ciencias sociales (Hayek, 1979a, pp.20-21). Según Hayek, las ciencias sociales estudian las acciones conscientes de los hombres; acciones racionales consistentes en elegir varios cursos de acción de entre diferentes alternativas. Estas acciones no pueden ser estudiadas, sin embargo, apelando a reducciones materialistas consistentes en buscar en los estímulos externos la respuesta al por qué de dichas acciones, como hace el conductismo al negar el conocimiento derivado de la introspección. Esto es así porque el objeto de estudio de las ciencias sociales es el conjunto de opiniones que dan sentido a la acción de los agentes; opiniones que obviamente no pueden ser contrastadas por métodos naturalistas. ${ }^{5}$ Además, según Hayek, nosotros conocemos las acciones de los otros y por ende es posible la ciencia social; porque comprendemos lo que se comporta igual a nosotros:

"The points which we want to stress are that in all such attempts we must start from what men think and mean to do: from the fact that the individuals which compose society are guided in their actions by a classification of things or events according to a system of sense qualities and of concepts which has a common structure and which we know because we, too, are men [...]" (57).

\footnotetext{
${ }^{5}$ Hayek, 1979a, p. 47. Esta concepción es fundamental para el análisis que hace Hayek de la economía. La actividad económica no puede ser definida en términos objetivos; sino sólo con referencia a propósitos.
} 

gnoseológica de la crítica a la economía planificada

Hayek en "Scientism" adelantará un punto interesante que luego será retomado en The Sensory Order; a saber, que nuestras representaciones sensoriales de las cosas no coinciden con las cosas que realmente existen en el mundo. Esto es así porque el cerebro actúa como un sistema clasificatorio, de modo tal que las cualidades que percibimos son efecto de una determinada clasificación que nuestra mente ha hecho. De esto se desprende, concluye Hayek, que pueda ser que diferentes objetos tengan iguales efectos sensoriales en distintas personas; o al revés, que objetos iguales tengan efectos sensoriales distintos en diferentes personas. ${ }^{6}$ La idea de que nuestras representaciones no coinciden con la realidad no es indiferente para el Hayek liberal; en tanto que utilizará esta concepción gnoseológica para criticar la planificación en economía. Hayek sostiene que tratar a los objetos de la actividad humana en términos de sus atributos "reales", en vez de las opiniones subjetivas que tienen dichas personas de dichos objetos y derivadas de las representaciones fruto de la organización de las sensaciones por parte de la mente, lleva a un grave error; a saber, concebir a la sociedad como pudiendo ser dirigida por una mente suprema con conocimiento absoluto que haga innecesario el interés del estudioso de las ciencias sociales por las opiniones y representaciones de los agentes. Así, conceptos como "posibilidades objetivas de producción" (que sirven para algunos críticos del sistema de libre mercado para sostener que el libre mercado deja recursos sin utilizar y por ende es ineficiente) le parecen a Hayek absurdos. Dichos conceptos no se basan en las opiniones y expectativas subjetivas de los agentes económicos y, por ende, no son válidos para ser enunciados por las ciencias sociales. Pues, éstas, como se ha visto, se ocupan de las representaciones, opiniones y expectativas subjetivas de los agentes. Si las representaciones no coinciden con la realidad necesariamente, no es posible decir que el sistema económico deja "efectivamente" recursos sin utilizar. El supuesto central de Hayek en este punto es que aquellos que utilizan tales conceptos que van más allá de las representaciones subjetivas suponen que el conocimiento disperso puede llegar a alcanzarse por una sola mente, la cual además obligaría a todos los miembros de la sociedad a actuar según su entender (Hayek, 1979a, pp.90-91). Un ejemplo concreto servirá para ilustrar este punto. Supongamos que en un país determinado, la frontera de posibilidades de producción del trigo es de diez millones de toneladas en un mercado de competencia perfecta y en el sistema de libre mercado (esto supone que, dada la competencia perfecta y el libre mercado, no se dejan recursos ociosos). Ahora bien, el gobierno de dicho país, no muy creyente de las bondades del libre mercado, sostiene que se están dejando recursos escasos; recursos que podrían dar más trabajo y generar más riqueza. Así, actúa en consecuencia con una serie de medidas intervensionistas regulando la actividad económica. Hayek, ante esto, diría: "El supuesto del gobierno es que sabe más que la suma del conocimiento de todos los agentes económicos. Esto es un error, pues el conocimiento es disperso y nadie puede arrogarse la facultad de

\footnotetext{
${ }^{6}$ Se sugiere consultar Hayek, 1979a, pp.80-84. En "La Primacía de lo abstracto", Hayek también sostiene la idea de un orden mental clasificatorio (Hayek, 1981, pp.32, 34, 37).
}

Revista Filosofía UIS, Vol. 15, No. 1, enero-junio de 2016 pp. 89-105 
tener todo el conocimiento sobre la actividad económica. Y mucho menos, bajo esa supuesta facultad, conducir las decisiones de los demás".

\section{La teoría del conocimiento en The sensory order}

Bruce Caldwell comenta que Hayek interrumpió su proyecto sobre el abuso de la Razón para escribir un libro con caracteres marcadamente académicos y científicos: The Sensory Order. La razón de esto es que Hayek quería ganar reputación científica y no ser visto simplemente como un propagandista político (Caldwell, 2004, p.257). The Sensory Order versa sobre Psicología y lo que hoy llamaríamos Filosofía de la Mente y es, según el mismo Hayek, su libro más "indigerible y aburrido", dada su complejidad en relación con el resto de la obra del economista austriaco y debido a su hincapié en la descripción de los procesos mentales. ${ }^{7}$ Hayek, en The Sensory Order, establece una diferenciación entre dos órdenes de cosas: 1) el orden físico, que se revela mediante la ciencia natural y 2) el orden mental, u orden sensorial, orden que clasifica los objetos externos de cierta manera. Lo interesante que nos dice Hayek es que no existe una correspondencia directa entre el orden que se presenta en las cosas existentes en el mundo (orden físico) y la clasificación que nosotros como individuos cognoscentes hacemos de las representaciones que nos acaecen en contacto con el mundo (Hayek 1992, pp.3-4). De este modo, el mundo físico y las representaciones que tenemos de él no necesariamente coinciden:

In so far as the similarities or differences of the phenomena as perceived by us do not correspond with the similarities or differences which the perceived events manifest in their relation to each other, we are not entitled to assume that the world appears to us as it does because it is like that [...] (6).

Así, la tarea de la psicología teórica será explicar cómo es que el orden sensorial difiere del físico. Para sostener esta carencia de isomorfía entre el orden físico y el sensorio, Hayek apela, entre otros argumentos, a la teoría de la energía específica de los nervios de Johannes Müller; quien sostiene que no son las propiedades físicas de los estímulos los que determinan la cualidad de las sensaciones resultantes; sino alguna propiedad de los impulsos internos. ${ }^{8}$

\footnotetext{
Hayek, 1979b, p.199. El interés de Hayek por la psicología descriptiva y experimental comienza ya en los primeros años de sus estudios universitarios. Más aún, estando en Suiza, en sus años de estudiante, Hayek trabajó un par de semanas con el fisiólogo del cerebro Constantin Von Monakow, recibiendo influencias de Ernst Mach y de Koffka (Caldwell, 2004, pp.136- 137).

${ }^{8}$ Hayek, 1992, p.11. La teoría de la Gestalt sostenía un marcado isomorfismo entre los órdenes físicos y sensorios, contra lo que Hayek argumentará a lo largo de The Sensory Order (1992. pp.37-38).
} 
Ahora bien, ien qué consiste esta llamada "propiedad de los impulsos internos" que hace que dados los mismos estímulos no se presenten en diferentes personas afecciones que den lugar a ordenes sensorios similares? La respuesta central de Hayek a esta pregunta es que el sistema nervioso humano es un gran mecanismo de clasificación. De este modo, todos los estímulos que afectan al sujeto son clasificados por el sistema nervioso, que funciona como un procesador categorial de estímulos, ordenándolos según corresponda en el esquema cognitivo. Pero ¿en qué consiste este proceso de clasificación? Hayek responderá que tanto en el desarrollo filogenético como en el ontogenético, las especies y los individuos sufren determinadas experiencias y experimentan ciertos aprendizajes; aprendizajes y experiencias que, dado un estímulo nuevo, sirven como condición de posibilidad de clasificación de los nuevos estímulos que aparecen. Es decir, la idea central de Hayek es que la mente humana organiza los estímulos nuevos de acuerdo a cómo se pueden relacionar e insertar en el sistema de experiencias y aprendizajes previos (1992, pp.53 y 105). A estos aprendizajes y experiencias previas los Ilama Hayek "pre-sensoriales":

One important consequence of this relation between physiological linkages and sensory experience is that there will be implicit in all sensory experience certain relations determined by earlier linkages...which have never been the object of sensory experience in the ordinary meaning of this term [...] (106).

De este modo, las percepciones sensoriales están sujetas a un acto de clasificación que depende de anteriores vínculos mentales que ya están en el cerebro. El mismo Hayek considera a la percepción como un acto de interpretación:

\begin{abstract}
All we can perceive of external events are therefore only such properties of these events as they possess as members of classes which have been formed by past "linkages". The qualities which we attribute to the experienced objects are strictly speaking not properties of that objects at all, but a set of relations by which our nervous system classifies them [...] (143).
\end{abstract}

\title{
5. The sensory order: anticonductismo e ideas aprioristas
}

Para Hayek, como hemos visto anteriormente, cada sensación debe ser considerada como una interpretación de un evento a la luz de la experiencia pasada no sólo de los individuos sino de las especies. ${ }^{9}$ Así, esta idea de una mente que clasifica las sensaciones que tenemos según ciertas categorías podría ser considerada como neokantiana (166). Pero más allá de ver si Hayek es o no neokantiano, lo cierto es que The Sensory Order es netamente anticonductista. El programa de investigación conductista es asociacionista; es decir, sostiene que nuestro conocimiento se basa pura y exclusivamente en nuestras sensaciones y que dichas representaciones nos acaecen en nuestra mente de la misma forma

${ }^{9}$ Hayek, 1992, p.166. 
en la cual funcionan en la realidad: "la estructura de la conducta sería una copia isomórfica de las contingencias o covariaciones ambientales" (Pozo, 1992, pp.2526). Hayek, por otro lado, como hemos visto, sostendrá la falta de isomorfía entre nuestras representaciones mentales y el orden del mundo.

Clarence Irving Lewis, en Mind and the World-Order sostendrá que por lo general el paradigma de lo a priori es la definición, siendo ésta no falsable: "Si la experiencia fuera distinta de lo que es, la definición y su correspondiente clasificación podría ser inconveniente, inútil o fantástica, pero no podría ser falsa. La mente hace clasificaciones y determina significados; al hacerlo crea aquella verdad sin la cual no podría haber otra verdad" (Duran, Di Gregori, 2003, p. 98). Hayek sostendrá ideas que uno podría tal vez poner en analogía con Lewis en The Sensory Order; a saber, que el conocimiento no puede ser nunca contradicho por experiencias sensoriales. Como todo nuestro conocimiento se debe a la experiencia, debe contener elementos que no pueden ser contradichos por la experiencia; es decir, la experiencia sensorial presupone siempre un orden sensorial que precede a la experiencia misma y a la cual no puede contradecir sin minar su propia validez (si una experiencia sensorial " $\mathrm{x}$ " se funda en determinado orden mental " $y$ ", no podría contradecir a " $y$ " sin minar sus fundamentos). ${ }^{10}$

\section{Individuos diferentes y la fundamentación de la teoría del valor subjetivo}

En este apartado quiero presentar la siguiente hipótesis que a nuestro juicio resulta sumamente interesante: Quizá las concepciones gnoseológicas de Hayek dan lugar a la posibilidad de fundamentación de la teoría del valor subjetivo de la escuela neoclásica. Una de las diferencias del programa de investigación clásico y el neoclásico en Economía está dada por las concepciones diferentes

\footnotetext{
${ }^{10}$ Hayek, 1992, pp. 167, 169, 172. Hayek, en consonancia con esto sostiene que la ciencia tiende necesariamente hacia un estado último en el cual todo conocimiento está encarnado en las definiciones de los objetos con los cuales está relacionado; y en el cual todo enunciado verdadero con respecto a estos objetos es por tanto analítico y tautológico y no puede ser falsado por la experiencia (171). Teniendo esto en mente, uno podría sostener que Hayek ha cambiado su postura epistemológica desde el falsacionismo de "Economics and Knowledge" a un apriorismo quizá radical en The Sensory Order. Esta postura es, sin embargo, controvertida. Bruce Caldwell ni siquiera estaría de acuerdo en sostener que Hayek pasa de un apriorismo miseano a una postura falsacionista popperiana en "Economics and Knowledge" (Caldwell, 2004, pp.337). Y aún otros no estarían de acuerdo en sostener que The Sensory Order es una vuelta al apriorismo de Mises. Más bien, The Sensory Order estaría enmarcado en una posición Epistemológica Evolutiva, cuyo apriorismo se diferenciaría claramente de Mises. Esto, sin embargo, merece mayor atención y deberá ser estudiado ulteriormente con más detenimiento (Agradezco los comentarios del Dr. Gabriel Zanotti sobre la posibilidad de interpretar The Sensory Order como enmarcado en una Epistemología Evolutiva).
} 
concernientes a la teoría del valor. En breves y simples palabras, mientras los clásicos (Smith, Malthus, Ricardo, Marx) definían la riqueza como una magnitud objetiva que se derivaba fundamentalmente del trabajo aplicado a la producción de dicha riqueza, los neoclásicos (a partir de la llamada "revolución marginalista" con Jevons, Walras, Menger, Marshall, Pareto, etc.) sostenían que la medición de la riqueza es absolutamente personal. Es decir, la riqueza de dos personas es incontrastable porque depende únicamente del punto de vista subjetivo de cada persona. Por ejemplo, en este marco conceptual, uno no podría establecer que una persona que vive en EE.UU. y tiene un capital de \$1.000.000 es más rica que un mendigo que perciba alrededor de $\$ 200$ mensuales y no tenga capital alguno. Pues, diría la teoría neoclásica del valor subjetivo, las necesidades y preferencias de ambos individuos podrían ser disímiles y sólo ellos son los que subjetivamente pueden medir su riqueza, en cuanto a si están o no conformes con lo que perciben. Así, ninguna política con aires de redistribución tendría sustento teórico porque desde la teoría del valor subjetivo se nos preguntaría: ¿qué derecho tiene el Estado a establecer los cánones de objetividad de lo que consideramos "riqueza", de tal modo que se justifique la redistribución del ingreso?

Si bien es cierto que Hayek no intenta de ningún modo llevar las conclusiones gnoseológicas de The Sensory Order al campo de la fundamentación de la teoría del valor subjetivo, bien vale la pena plantearse la hipótesis de si es posible fundar la teoría del valor subjetivo en las concepciones gnoseológicas de Hayek. Veamos con más detenimiento lo que nos proponemos. A lo largo de The Sensory Order, Hayek trata de mostrar la imposibilidad de que haya dos individuos con características mentales iguales. Por ejemplo, en el Capítulo V, que versa sobre la estructura del orden mental, Hayek sostiene que ya que la estructura mental del sistema nervioso se modifica por cada nueva recepción de estímulos externos y éstos estímulos a su vez interactúan con el orden sensorial pre-existente, no puede haber dos individuos iguales en sentido mental porque a) no reciben los mismos estímulos y b) no tiene el mismo orden sensorio pre-existente: "Because it is the whole history of the organism which will determine its action [...]" (Hayek, 1992, p.123, énfasis del autor).

Ahora bien, se podría objetar la hipótesis que intentamos exponer aquí diciendo que quizá Hayek en The Sensory Order no nos está diciendo más que una verdad de perogrullo (que no todos tenemos las mismas vivencias y que nuestra mente no clasifica las cosas de la misma forma, para decirlo en términos simples) que no tiene intención ni podría fundamentar la teoría subjetiva del valor. Aún cuando esta crítica esté en lo cierto, todavía restaría ver qué papel juega el artículo "Scientism" y las concepciones epistemológicas hayekianas concernientes a la Epistemología de las ciencias sociales. Si el interés del estudioso de las ciencias sociales son las opiniones y representaciones de los agentes; entonces la teoría del valor subjetivo sería la única teoría del valor aceptada dentro de esta concepción acerca del rol y alcance de las ciencias sociales, ya que no podríamos saber qué 
es el "valor" o la "riqueza", independientemente del juicio de los agentes. Sin embargo, Hayek no intenta este camino de fundamentación de la teoría del valor subjetivo al menos en los textos estudiados, ni parece haber indicios en la obra de Hayek de un planteo de fundamentación análogo; que quizá ya sea dado por descontado por el mismo Hayek, dadas sus críticas al objetivismo y su defensa de posturas subjetivistas en "Scientism".

\section{Conclusiones}

El objetivo planteado para este trabajo fue mostrar cómo las concepciones del conocimiento en Hayek le son útiles para elaborar una crítica teórica de la economía planificada. Para llevar a cabo este estudio se trabajó con tres grupos de textos pilares en la literatura gnoseológica Hayekiana: 1) "Economics and Knowledge" y "The use of knowledge in Society"; 2) "Scientism" y 3) The Sensory Order.

En "Economics and Knowledge" y "The use of knowledge in Society" se vio cómo Hayek sostuvo el carácter disperso del conocimiento económico, la no omnisciencia de los agentes económicos y la no isomorfía entre los hechos económicos y las representaciones que los agentes tienen de los hechos económicos. Así, como el conocimiento está disperso y las decisiones económicas se basan en el conocimiento "aquí y ahora" de ciertas informaciones económicas particulares, ningún Estado puede pretender coordinar la actividad económica sobre la base de un conocimiento que no posee de ningún modo.

En "Scientism", la crítica de Hayek a la economía planificada se basa además en sostener que el supuesto inherente a justificar un manejo centralizadoplanificado de la economía es que la ciencia económica trata de atributos reales del funcionamiento del mundo; mientras que en realidad se trata del estudio de las representaciones de los agentes y cómo dichas representaciones influyen o no en la coordinación económica de los recursos y preferencias individuales.

Por último planteamos la posibilidad de una fundamentación de la teoría del valor subjetivo (con su consiguiente crítica a las políticas centralizadas redistribucionistas) en ciertos puntos centrales que pueden derivarse de "Scientism" y The Sensory Order; a saber, la imposibilidad de la isomorfía entre ordenes sensoriales de distintos individuos y el carácter subjetivista del abordaje teórico de las ciencias sociales.

Como analizamos en páginas anteriores, Hayek sigue a Popper al sostener que la teoría económica tiene un elemento empírico que es pasible de ser falsado; esto es, las proposiciones empíricas basadas en cómo el conocimiento es adquirido y comunicado. El falsacionismo es, además, para Popper, un criterio de demarcación para distinguir qué campo de conocimiento es científico y cual no. En La Lógica de 
la Investigación Científica, Popper sostiene que su criterio de demarcación es una propuesta para un acuerdo o convención:

En cuanto a si tal convención es apropiada o no lo es, las opiniones pueden diferir; mas sólo es posible una discusión razonable de estas cuestiones entre partes que tienen cierta finalidad común a al vista. Por supuesto que la elección de tal finalidad tiene que ser, en última instancia, objeto de una decisión que vaya más allá de toda argumentación racional...Así pues, admito abiertamente que para llegar a mis propuestas me he guiado, en última instancia, por juicios de valor y por predilecciones. Más espero que sean aceptables para todos los que no sólo aprecian el rigor lógico, sino la libertad de dogmatismos [...] (1985, p. 37).

Por otro lado, en El desarrollo del Conocimiento Científico. Conjeturas y Refutaciones, Popper claramente deja en claro sus juicios de valor: él quiere que sea ciencia un conjunto de conocimientos a los cuales sea posible falsar, evitando así caer en dogmatismos cerrados y verdades totalizadoras. Pero no sólo eso, él aboga por una sociedad en donde se puedan discutir libremente las ideas y en donde prime una teoría liberal no sólo de la libre discusión crítica sino también en un plano político más abarcador. ${ }^{11}$

Ante estas ideas de Popper la pregunta es: ¿sus concepciones políticas y valores liberales determinan su criterio de lo que es Ciencia, es su concepción gnoseológica la que determina sus valores políticos y liberales o son independientes dichos campos? Con respecto a Hayek podemos hacer una pregunta análoga: ¿son sus concepciones gnoseológicas las que lo llevan a criticar a la economía planificada o son más bien sus intereses de criticarla los que lo llevan a elaborar una teoría del conocimiento que sirva para dicha crítica? En el caso de Popper la respuesta trae polémica y se han dedicado varios estudios a tal tema. En el caso de Hayek la pregunta sin duda trae polémica. No sólo por la amistad que unió a ambos autores; sino porque, como se ha dicho en la Introducción, Hayek en sí mismo es un autor que ha traído y trae aún todavía polémica $\varphi$

\section{REFERENCIAS}

Caldwell, B. (2004). Hayek's Challenge: An Intelectual Biography of F.A Hayek. Chicago \& London: The University of Chicago Press.

Connin, L. (1990). "Hayek, Liberalism and Social Knowledge". Canadian Journal of Political Science, 23 (2).

\footnotetext{
${ }^{11}$ Popper, 1967, pp 43-52, 405-409. Obviamente, no hay que olvidar sus críticas al Marxismo teórico y al sistema socialista en "La Miseria del Historicismo" y "La Sociedad abierta y sus enemigos".
} 
Gómez, R. (2003). Neoliberalismo Globalizad: Refutación y debacle. Buenos Aires: Macchi.

Hausman, D. (1992). The inexact and separate science of economics. New York: Cambridge University Press.

Hayek, F. (1967). Studies in Philosophy, Politics, and Economics. Chicago: University of Chicago Press.

Hayek, F. (1979a). The Counter-Revolution of Science. Indianapolis: Liberty Press.

Hayek, F. (1979b). The political Order of a Free People. Vol. 3 of Law, Legislation, and Liberty. Chicago: The University of Chicago Press.

Hayek, F. (1980a). Individualism and Economic Order. Chicago: The University of Chicago Press.

Hayek, F. (1980b). Individualism and Economic Order. Chicago: The University of Chicago Press.

Hayek, F. (1981). Nuevos Estudios en Filosofía, Política, Economía e Historia de las ideas. Buenos Aires: Eudeba.

Hayek, F. (1992). The Sensory Order. Chicago: The University of Chicago Press.

Hayek, F. (1994). Hayek on Hayek: An Autobiographical Dialogue. Chicago: The University of Chicago Press

Hayek, F. (2005). Camino de servidumbre. Barcelona: Alianza editorial.

Horwitz, S. (2000). From The Sensory Order to the liberal order: Hayek's nonrationalist Liberalism. Review of Austrian Economics, 13 (1).

Hutchison, T. (1981). The Politics and Philosophy of Economics: Marxians, Keynesians, and Austrians. Oxford: Blackwell.

Duran, C. y Di Gregori, C. (2013). "La naturaleza de lo a priori y el elemento pragmático en el conocimiento" de Clarence Irving Lewis. Redes, 10 (20), 89-117. Recuperado de < http://www.redalyc.org/articulo.oa?id=90702005>

Lawson, T. (2005). "The (confused) state of equilibrium analysis in modern economics: an explanation". Journal of Post-Keynesian Economics, 27 (3). 

gnoseológica de la crítica a la economía planificada

Luján, C. (2003). "El apriorismo de Ludwig von Mises". Laissez-Faire, (18-19), $37-56$.

Ortiz, D. (2009). "El orden sensorial, individualismo y conocimiento económico en la obra de F.A. Hayek". Revista de economía institucional, 11 (20).

Popper, K. (1967). El desarrollo del Conocimiento Científico: Conjeturas y Refutaciones. Buenos Aires: Paidós

Popper, K. (1985). La Lógica de la Investigación Científica. Buenos Aires: REI.

Pozo, J. (1992). Teorías Cognitivas del aprendizaje. Madrid: Morata.

Rosicka, J. (2002). "Liebniz's Science on Economy. Economic Security versus Economic Scarcity". En Actas del VII Internationaler Leibniz-Kongress. Nihil Sine Ratione, Hannover.

Scarano, E. (2004). "El Apriorismo de Ludwig Von Mises". Revista Libertas, (41).

Yeager, L. (1994). Mises and Hayek on Calculation and Knowledge. The Review of Austrian Economics, 7 (2). 